\title{
Efecto de la formulación de galletas en la secreción de hormonas de saciedad
}

\section{Effect of biscuit formulations in the release of satiety hormones}

\author{
Martinez-Saez, Nuria (1), Hochkogler, Christina Maria (2), Somoza, Veronika (2), del Castillo, María Dolores (1) \\ (1) CIAL, Instituto de Investigación de Ciencias de la Alimentación (UAM-CSIC), Madrid, España \\ (2) Departamento de Química Nutricional y Fisiológica, Facultad de Química, Universidad de Viena, Austria.
}

Contacto: mdolores.delcastillo@csic.es

RECIBIDO: $13 / 4 / 2016$ - APROBADO: $13 / 6 / 2016$

\begin{abstract}
Resumen
El objetivo de esta investigación fue desarrollar una nueva formulación de galleta con bajo índice glicémico y elevada calidad sensorial para el control efectivo de la saciedad. Se emplearon como ingredientes stevia (edulcorante hipocalórico), fructooligosacáridos (FOS), para mejorar la tolerancia a carbohidratos y calidad sensorial del alimento, y fibra dietética antioxidante de café (marros de café). Los alimentos e ingredientes individuales se digirieron in vitro simulando condiciones de digestión humana. La liberación in vitro de péptido similar al glucagón tipo 1 (GLP1) y serotonina se estimuló incubando células humanas intestinales, HuTu-80 y Caco-2, con digeridos de galletas $(0,5$, 0,05 y $0,01 \mathrm{mg} / \mathrm{ml}$ ) durante $90 \mathrm{~min}$ y $5 \mathrm{~min}$, respectivamente. La cuantificación de las hormonas GLP1 y serotonina se realizó por ELISA. La secreción de hormonas en células tratadas con digeridos de galletas tradicional y nueva fueron del mismo orden de magnitud ( $\mathrm{p}>0,05)$. La fibra antioxidante del café estimuló la secreción de hormonas saciantes mientras que la stevia resultó inefectiva. La sustitución de sacarosa por stevia no afectó la capacidad saciante de las galletas. En conclusión, se ha logrado un nuevo alimento con alto contenido en fibra antioxidante, saludable, saciante, de buena calidad sensorial apto para individuos sanos y diabéticos.

Palabras clave: Alimento de bajo índice glicémico, fibra antioxidante de café, FOS, GLP-1, serotonina, stevia.
\end{abstract}

\begin{abstract}
This research aimed to evaluate the potential of a new biscuit formulation with low glycaemic index and high sensorial quality for an effective control of satiety. Stevia as hypocaloric sweetener, fructooligosaccharides (FOS) as enhancer of glucose tolerance and sensorial quality, and antioxidant insoluble dietary fibre (spent coffee grounds) were used as food ingredients. Food and individual ingredients were digested in vitro by mimicking human oral-gastrointestinal digestion. The in vitro release of glucagon-like petide-1 (GLP-1) and serotonin was stimulated by incubation of human intestinal cells, HuTu-80 and Caco-2, with digests at final concentrations of 0,5, 0,05 and $0,01 \mathrm{mg} / \mathrm{ml}$, for $90 \mathrm{~min}$ and $5 \mathrm{~min}$, respectively. Quantification of the hormones called as GLP1 and serotonin was carried out by ELISA. The traditional and new biscuits induced the release of hormones in the same order of magnitude $(p>0,05)$. Antioxidant coffee fibre contributed to stimulate the release of satiety hormones while stevia was ineffective. Replacement of sucrose by stevia did not affect the satiating capacity of the biscuit. In conclusion, a new healthy food containing high level of antioxidant dietary fibre, satiating, good sensorial quality for healthy people and friendly for diabetics has been obtained.
\end{abstract}

Keywords: Antioxidant coffee fibre, FOS, GLP-1, low glycaemic index, serotonin, stevia.

\section{Introducción}

La obesidad es uno de los principales problemas de salud pública del siglo XXI en todo el mundo. En 2014, más de 1.900 millones de adultos mayores de 18 años tenían sobrepeso, de los cuales más de 600 millones eran obesos (World Health Organization, 2014). La mayoría de la población mundial vive en países donde el sobrepeso y la obesidad se cobran más vidas humanas que la insuficiencia ponderal (World Health Organization, 2014). Enfermedades como diabetes mellitus tipo 2, hipertensión, enfermedades cardiovasculares y varios tipos de cáncer están asociadas con la obesidad (Nutritional Heart, Lung and Blood Institute, 2013).

Una alimentación saludable puede considerarse como estrategia sostenible para reducir el riesgo de obesidad y las enfermedades relacionadas tales como la diabetes tipo 2. Así, la búsqueda de nuevos ingredientes y alimentos con capacidad de controlar de forma efectiva los procesos de saciedad y de glicemia postprandial es hoy en día una línea de investigación prioritaria. 
La stevia, en concreto los glucósidos de esteviol, poseen efectos beneficiosos en la salud como efecto hipocalórico, antioxidante y anti-diabético (Sharma, et al., 2012). Los fructooligosacáridos (FOS) presentan carácter prebiótico (Kumar, et al., 2012) y capacidad de reducir la respuesta glicémica postprandial (EFSA, 2014). Otro ingrediente esencial en la formulación de alimentos para el control del peso corporal y la diabetes es la fibra dietética. Los subproductos agroalimentarios y en particular los del café son una fuente abundante y sostenible de varios componentes bioactivos beneficiosos para la reducción de riesgo de patologías crónicas, incluyendo fibra antioxidante (del Castillo, et al., 2014).

Las hormonas serotonina y péptido similar al glucagón tipo 1 (GLP-1), secretadas en el tracto gastrointestinal, están implicadas en los procesos de saciedad. La ingesta de ciertos alimentos e ingredientes activa señales de saciedad en el intestino que implican la liberación de serotonina y GLP-1, la activación de sus receptores gastrointestinales y la señalización aferente vagal, y por lo tanto, la regulación del apetito en el ser humano (Mawe y Hoffman, 2013; Van Bloemendaal, et al., 2014). La hormona GLP-1 además de estimular la saciedad mejora la glicemia vía secreción de insulina inducida por glucosa en las células beta pancreáticas e inhibe la liberación de glucagón (Lee, 2016).

El objeto de esta investigación es lograr una nueva formulación de snack saludable apto para personas sanas interesadas en prevenir patologías crónicas metabólicas y diabéticos, sin renunciar a su calidad sensorial. El estudio tiene como objetivo además obtener información novedosa en relación al efecto de ingredientes innovadores que se emplean en la elaboración de los alimentos para personas con requerimientos nutricionales particulares (control del peso corporal) y con fines médicos (prevención y tratamiento de enfermedades crónicas metabólicas tales como diabetes) sobre la secreción de serotonina y GLP-1. En nuestro conocimiento no existen estudios previos del efecto de la fibra antioxidante de café (marros del café) y sus productos de digestión sobre la secreción de estas hormonas. Los estudios publicados hasta la fecha en relación a los efectos saciantes de stevia son muy escasos y de gran interés, teniendo en cuenta el rápido incremento de su popularidad en todo el mundo.

\section{Materiales y Métodos}

El plan de trabajo propuesto en este estudio se presenta en la Figura 1. Tal como queda reflejado en el esquema, se prepararon galletas a base de harina de trigo empleando como edulcorantes sustitutos del azúcar, maltitol y stevia, y como nueva fuente natural de fibra antioxidante de café un abundante subproducto de su procesado, marros de café (MC) (del Castillo, et al., 2014). Con el fin de obtener formulaciones de elevada calidad nutricional y sensorial, de acuerdo a las preferencias de los consumidores $y$ con potencial para reducir el riesgo de enfermedades crónicas como la obesidad y la diabetes, se llevó a cabo en primer lugar un análisis sensorial de las galletas propuestas. Se obtuvieron fracciones bioaccesibles de estos nuevos alimentos e ingredientes digeridos mimetizando las condiciones fisiológicas humanas de la digestión oral gastrointestinal. Los efectos de estos digeridos sobre la liberación de hormonas intestinales, serotonina y GLP-1, implicadas en procesos de saciedad y de glucemia postprandial en líneas celulares intestinales (Caco-2 y HuTu-80, respectivamente), fueron evaluados. En la Figura 1 se describen los materiales y métodos empleados para la instrumentación del plan de trabajo.
PREPARACIÓN DE GALLETAS

\section{Glucósidos de esteviol (STEVIA) \\ Edulcorante hipocalórico natural \\ Oligofructosa (OF) \\ Fibra soluble mejoradora del sabor \\ INGREDIENTES \\ ALIMENTARIOS INNOVADORES}

\section{Marros de café (MC)}

Fibra dietética insoluble antioxidante

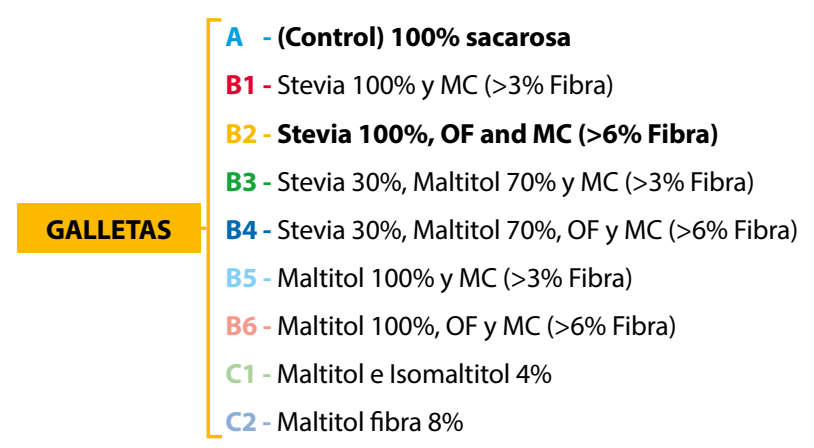

DIGESTIÓN ORAL GASTROINTESTINAL IN VITRO

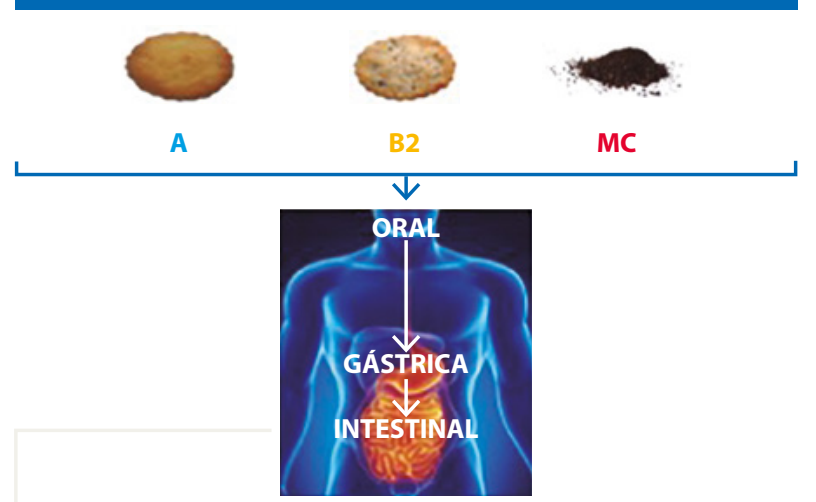

FRACCIÓN

INSOLUBLE

DIGERIDOS
FRACCIÓN

BIOACCESIBLE

\section{Colestiramina}

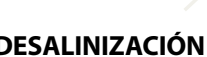

(Simulación reabsorción sales biliares)

EFECTO IN VITRO DE DIGERIDOS EN CÉLULAS INTESTINALES

Incubación con digeridos desalinizados y stevia $(0,01,0,05,0,5 \mathrm{mg} / \mathrm{ml})$

Células Caco-2

(Células epiteliales de

cáncer colorectal)

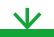

5 ' en oscuridad

SEROTONINA Células HuTu-80 (Células epiteliales de cáncer duodenal) $v$

$1 \mathrm{~h}$ ayuno $+1 \mathrm{~h} 30^{\prime}$

DIGERIDOS DESALINIZADOS

CUANTIFICACIÓN DE HORMONAS (ELISA)
Figura 1. Plan de trabajo metodológico. 


\begin{tabular}{|c|c|c|c|c|c|c|cc|}
\hline Ingredientes $\mathbf{( g )}$ & A & B1 & B2 & B3 & B4 & B5 & B6 \\
\hline Harina de trigo & 56 & 61,54 & 59,4 & 55,11 & 55,5 & 52,83 & 53,4 \\
Agua & 20 & 21,98 & 21,2 & 19,68 & 18,2 & 18,87 & 17,5 \\
Aceite de girasol & 7,75 & 8,52 & 8,2 & 7,63 & 7,1 & 7,31 & 6,8 \\
Levadura química & 0,55 & 0,60 & 0,58 & 0,54 & 0,5 & 0,52 & 0,48 \\
Sal & 0,37 & 0,41 & 0,39 & 0,36 & 0,34 & 0,35 & 0,32 \\
Lecitina de soja & 0,33 & 0,36 & 0,35 & 0,32 & 0,3 & 0,31 & 0,29 \\
Sacarosa & 15 & 0 & 0 & 0 & 0 & 0 & 0 \\
Maltitol & 0 & 0 & 0 & 11,81 & 10,8 & 16,04 & 14,9 \\
Stevia & 0 & 2,20 & 2,1 & 0,60 & 0,56 & 0 & 0 \\
FOS & 0 & 0 & 3,5 & 0 & 3 & 0 & 2,9 \\
Marros de café & 0 & 4,40 & 4,2 & 3,94 & 3,6 & 3,77 & 3,5 \\
TOTAL & 100 & 100 & 100 & 100 & 100 & 100 & 100 \\
\hline
\end{tabular}

Tabla 1. Formulaciones de las galletas: A- (control) con sacarosa; B1- stevia 100\% y marros de café (MC) (>3\% Fibra); B2- stevia $100 \%$, fructooligosacáridos (FOS) y MC (>6\% Fibra); B3- stevia 30\%, maltitol 70\% y MC (>3\% fibra); B4- stevia $30 \%$, maltitol 70\%, OF y MC (>6\% fibra); B5- maltitol 100\% y MC (>3\% Fibra); B6- maltitol 100\%, OF y MC (>6\% fibra).

\section{Ingredientes alimentarios}

Para la formulación de galletas, todos los ingredientes básicos se compraron en supermercados especializados y certificados. La lecitina de soja de grado alimentario se obtuvo de Manuel Riesgo S.A. (España). El maltitol fue proporcionado por Helm Iberica S.A. La stevia, con un 3\% de glucósidos de esteviol, se adquirió en un supermercado local. Los FOS en formato polvo (ORAFTI ${ }^{\oplus}$ 95) fueron una donación de Beneo-Orafti.

Como fuente de fibra antioxidante de café se emplearon MC procedentes del proceso industrial de obtención del café soluble o instantáneo. Los granos de café utilizados para su elaboración fueron de especie Robusta. La materia prima fue proporcionada por Prosol S.A (España) y almacenada $-20^{\circ} \mathrm{C}$ hasta su uso.

\section{Preparación de alimentos}

Se elaboraron un total de seis nuevas formulaciones de galletas sin azúcares (B1, B2, B3, B4, B5, B6) y una galleta siguiendo la formulación tradicional y utilizando como edulcorante sacarosa que se empleó como control (A). Las formulaciones de los siete alimentos se indican en la Tabla 1. Las galletas se prepararon empleando como ingredientes básicos harina de trigo y aceite de girasol. No se incluyó huevo en las formulaciones y el azúcar se sustituyó por stevia y/o maltitol como edulcorantes hipocalóricos en las formulaciones B1-B6. Los MC se añadieron como fuente insoluble de fibra antioxidante y los FOS para mejorar la tolerancia a la glucosa, aportar fibra soluble, favorecer la salud intestinal (prebiótico) y mejorar la textura y el sabor de los nuevos alimentos con elevado contenido de fibra antioxidante de café y stevia.

La masa se preparó mezclando inicialmente sal, levadura, FOS y edulcorantes, y sobre esta mezcla seca se añadió agua mineral a temperatura ambiente, hasta conseguir una mezcla homogénea. La lecitina y el aceite de girasol se mezclaron aparte y posteriormente se añadieron a la mezcla acuosa. Finalmente, la harina junto con la fibra antioxidante de café se fue añadiendo gradualmente a la mezcla y se trabajó hasta obtener una masa homogénea, elástica y ligeramente pegajosa. La masa se dejó reposar durante 30 min y transcurrido este tiempo se moldeó en forma de galletas con guías de grosor 3-5 $\mathrm{mm}$ y moldes de $6,5 \mathrm{~mm}$ de diámetro. La superficie de las galletas se pinchó para evitar la formación de burbujas durante el horneado a $185^{\circ} \mathrm{C}$ durante $16 \mathrm{~min}$.

\section{Alimentos comerciales}

Dos tipos de galletas comerciales con alta adhesión a la marca por parte de los consumidores españoles ( $\mathrm{C} 1$ y C2) se adquirieron en un supermercado local. C1 y C2 presentaban en su formulación maltitol e isomaltitol como edulcorantes y fibra dietética en un contenido del 3,6\% y $8,3 \%$, respectivamente.

\section{Calidad sensorial}

Las galletas elaboradas según las nuevas formulaciones (B1, $\mathrm{B} 2, \mathrm{~B} 3, \mathrm{~B} 4, \mathrm{~B} 5, \mathrm{~B} 6)$ y las comerciales $(\mathrm{C} 1, \mathrm{C} 2)$ se sometieron a un test sensorial hedónico. La evaluación sensorial se realizó con la participación de 26 catadores no entrenados. Se evaluó el nivel de aceptación global de las galletas teniendo en cuenta el color, la textura y el sabor, empleando una escala hedónica verbal de 7 puntos (1- me disgusta mucho y 7-me gusta mucho). La media de la puntuación dada a cada formulación de galletas se determinó. La nueva formulación de galleta con un grado de aceptación equivalente a las comerciales se seleccionó para estudiar su efecto en la liberación de las hormonas GLP-1 y serotonina. 


\section{Digestión oral gastrointestinal in vitro}

Previo al estudio del efecto de liberación de hormonas in vitro, se llevó a cabo una digestión abiótica oral, gástrica e intestinal, según el procedimiento descrito por Hollebeeck et al. (2013), con ligeras modificaciones. Los digeridos obtenidos se centrifugaron y fueron separados en dos fracciones, sobrenadante y precipitado. Sobre la fracción bioaccesible (sobrenadante) se realizó la última etapa, eliminación de las sales biliares, con el fin de imitar la reabsorción de las mismas que tiene lugar en el intestino durante el proceso de digestión humano (Edwards y Slater, 2009). Los digeridos desalinizados se congelaron a $-80^{\circ} \mathrm{C}$ y posteriormente fueron liofilizados. Los ensayos se realizaron por triplicado.

\section{Cultivo celular: Caco-2 y HuTu-80}

Células epiteliales tumorales de colon humano, Caco-2, se cultivaron en Medio de Eagle Modificado por Dulbecco (DMEM) suplementado con suero fetal bovino (10\%), L-glutamina (2\%) y penicilina/estreptomicina $(1 \%)$ a $37^{\circ} \mathrm{C}$ y $5 \%$ de $\mathrm{CO}_{2}$. Las células se crecieron en placas de 12 pocillos hasta alcanzar confluencia aproximadamente tres días después. La diferenciación celular se obtuvo transcurridos 21 días con cambio de medio cada dos-tres días. Las células Caco-2 ya diferenciadas se emplearon para el estudio de liberación de la hormona serotonina.

Células epiteliales cancerosas de duodeno humano, HuTu-80, se cultivaron en Medio Esencial Mínimo (MEM) suplementado con suero fetal bovino (10\%), L-glutamina (2\%) y penicilina/estreptomicina $(1 \%)$ a $37^{\circ} \mathrm{C}$ y $5 \% \mathrm{CO}_{2}$. Las células se crecieron en placas de 24 pocillos hasta alcanzar confluencia en un día transcurrido. Las células HuTu-80 no se diferenciaron y 24 horas después de sembrarse estaban preparadas para utilizarse en los ensayos de liberación de la hormona GLP-1.

Ambas líneas celulares se subcultivaron tres-cuatro veces tras su descongelación antes de usarse, con el fin de recobrar el ratio normal de crecimiento.

\section{Ensayos de liberación de serotonina y GLP-1}

Las fracciones solubles recuperadas de los digeridos de la galleta tradicional (A), galleta elaborada según la nueva formulación (B2) y fibra antioxidante de café (I3) - que contienen los componentes bioaccesibles para su absorción y metabolismo- y stevia se emplearon para estimular la secreción de hormonas causantes de saciedad en los modelos celulares descritos en los párrafos anteriores, siguiendo la metodología que se describe a continuación.

\section{Estimulación y cuantificación de serotonina}

Las células Caco-2 se suplementaron con las fracciones bioaccesibles $(150 \mu \mathrm{l})$ en tres concentraciones diferentes $(0,5,0,05,0,01$ $\mathrm{mg} / \mathrm{ml}$ ), por duplicado. Previamente, se llevó a cabo un lavado de las células con tampón fosfato salino (PBS). La estimulación de las células se realizó por 5 minutos con agitación orbital. Los sobrenadantes se retiraron y guardaron en congelación hasta posterior cuantificación de serotonina. Se incluyó como control positivo cinamaldehído $5 \mathrm{mM}$, y como control negativo tampón. La cuantificación de la hormona serotonina liberada por las Caco-2 fue realizada siguiendo las indicaciones del kit de ensayo por inmunoadsorción ligado a enzimas (ELISA) de alta sensibilidad (cat-n ${ }^{\circ}$ EA630/96, de DLD Diagnostika $\mathrm{GmbH})$. Tres subcultivos diferentes se emplearon en cada set de análisis, que se realizaron por sextuplicado $(n=3 \times 6=18)$. Los resultados se expresaron como \% frente al control T/C [\%].

\section{Estimulación y cuantificación de GLP-1}

Las células HuTu-80 se sometieron a un período de ayuno de $1 \mathrm{~h}$ con medio libre de suero, glucosa y glutamina, y estimulación con las fracciones bioaccesibles $(300 \mu \mathrm{l})$ a 0,5, 0,05, $0,01 \mathrm{mg} / \mathrm{ml}$, glutamina $40 \mathrm{mM}$ (control positivo) y tampón (control negativo) durante $1 \mathrm{~h} 30 \mathrm{~min}$ a $37^{\circ} \mathrm{C}$. El experimento se llevó a cabo por duplicado. Tras el periodo de estimulación se colectaron los sobrenadantes y se conservaron en congelación hasta su posterior análisis. La cuantificación de GLP-1 se realizó mediante ELISA sándwich siguiendo las instrucciones del kit (Cat-no EZGLP1T-36K de EMD Millipore). Tres subcultivos diferentes se emplearon en cada set de análisis, que se realizaron por sextuplicado $(\mathrm{n}=3 \times 6=18)$. Los resultados se expresaron como \% frente al control T/C [\%].

\section{Análisis estadístico}

Los análisis estadísticos se realizaron mediante el empleo de SigmaPlot 11.0 (Systat Software Inc., California, USA). Los resultados se expresaron como la media \pm desviación estándar (SD) para todos los análisis, excepto aquellos relativos al cultivo celular, los cuales se representaron como la media \pm error estándar de la media (SEM). Los datos se analizaron mediante análisis de varianza (ANOVA) de una vía y las diferencias entre medias mediante el Test de Holm-Sidak a un nivel de significación de $\alpha=0,05$.

\section{Resultados y Discusión}

\section{Análisis sensorial}

Como se muestra en la Figura 2, las formulaciones de galletas preparadas con stevia (B2), stevia y maltitol (B4) y maltitol (B6), que contienen en todos los casos fibra antioxidante de café y FOS en su formulación, obtuvieron puntuaciones significativamente mayores $(\mathrm{p}<0,05)$ que las respectivas galletas sin FOS (B1, B3 y B5). Los compuestos bioactivos, cafeína y glucósidos de esteviol, presentes en el café y la stevia, respectivamente, aportan un sabor amargo que persiste en boca (Hellfritsch, et al., 2012), pudiendo afectar negativamente la calidad sensorial del alimento. El retrogusto de los esteviósidos en particular es muy persistente, lo que limita su uso a determinadas concentraciones en muchos alimentos. Los FOS presentan un ligero dulzor que mejora significativamente $(\mathrm{p}<0,05)$ la calidad sensorial de los alimentos elaborados incluyendo en su formulación stevia y fibra antioxidante de café (B2, B4 y B6) como ingredientes. La incorporación de FOS en las galletas tiene un impacto positivo en la calidad nutricional del alimento. A los FOS se le atribuyen carácter prebiótico (Kumar, et al., 2012), efecto fibra (Sartori da Silva, et al., 2007) y capacidad de reducir la respuesta glicémica postprandial (EFSA, 2014). Por tanto, los FOS son un ingrediente esencial de la formulación de las 
nuevas galletas, dado que su incorporación solventa simultáneamente problemas de calidad sensorial y mejora el valor nutricional del alimento. Su adición podría tener impacto no solo en el sabor sino también en la textura del producto final (Ishwarya y Prabhasankar, 2013).

El grado de aceptación global de las galletas que contienen FOS en su formulación (B2, B4 y B6) y las comerciales (C1 y $\mathrm{C} 2$ ), con alta adhesión del consumidor y similares características nutricionales en lo que se refiere al uso de edulcorantes hipocalóricos y el contenido de fibra incorporado, no difirió significativamente ( $p>0,05)$ (Figura 2). A las galletas B2, B4, $\mathrm{B} 6$ y C2 se les puede atribuir la alegación nutricional "alto contenido de fibra", de acuerdo con lo establecido en el Reglamento (CE) 1924/2006 del Parlamento Europeo (Unión Europea, 2006).

Los resultados del estudio indican que la combinación de los ingredientes fibra antioxidante de café, stevia y FOS resulta adecuada para la obtención de un snack saludable y destaca el interés de los FOS como ingrediente funcional, tanto desde el punto de vista tecnológico como nutricional. Teniendo en cuenta los resultados del análisis sensorial, se seleccionó la galleta B2, que contiene en su formulación fibra antioxidante de café, stevia y FOS, para realizar los estudios posteriores de liberación de hormonas (serotonina y GLP-1) implicadas en procesos de saciedad y tolerancia a la glucosa postprandial.

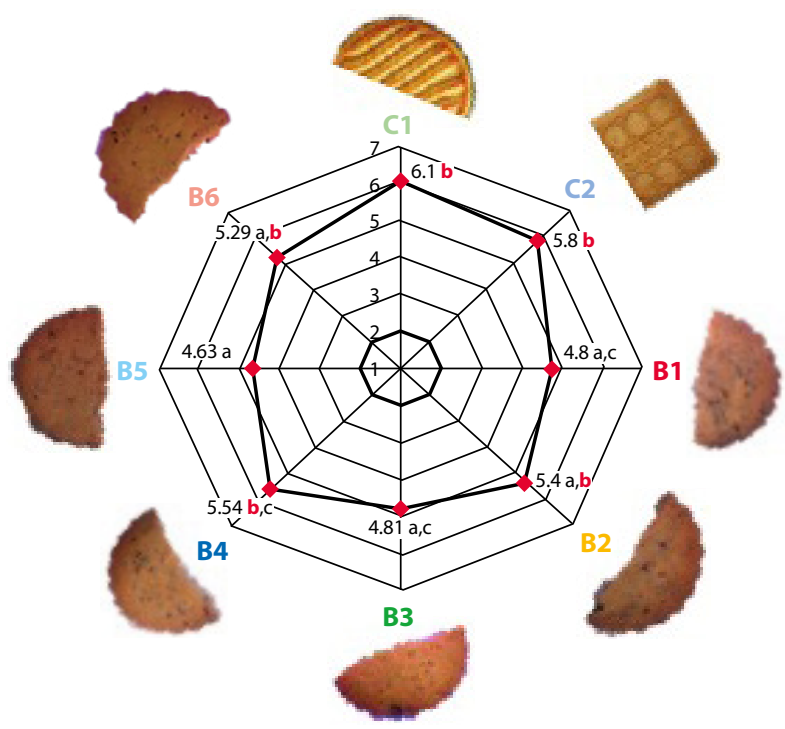

Figura 2. Diagrama de tela de araña con las puntuaciones medias $(n=26)$ de la escala de 7 puntos para la aceptación global de las galletas comerciales $(\mathrm{C} 1$ y $\mathrm{C} 2)$ y de las galletas reformuladas (B1, B2, B3, B4, B5 y B6) cuya composición se indica en Tabla 1. Letras diferentes denotan diferencias significativas en la calidad sensorial de los alimentos objeto de estudio $(\mathrm{p}<0,05)$.

\section{Liberación de serotonina}

Los compuestos bioaccesibles que se recuperaron tras la digestión oral gastrointestinal in vitro de la galleta con alto contenido en fibra y sin edulcorante calórico (B2) en una concentración de $0,5 \mathrm{mg} / \mathrm{ml}$ estimularon la secreción de la hormona serotonina en las células Caco-2 diferenciadas (Tabla 2). Los niveles de serotonina en las células tratadas con el nuevo alimento $(354,7 \pm 42,7 \%)$ fueron cuatro veces más elevados que los detectados en las muestras correspondientes a las células control (100\%). No se observaron diferencias significativas $(p>0,05)$ entre los niveles de secreción de serotonina detectados en células tratadas con los compuestos bioaccesibles de las galletas elaboradas según la nueva formulación y la tradicional, en la que se emplea sacarosa como edulcorante (A).

Los compuestos bioaccesibles que se liberaron durante el proceso de digestión de la fibra antioxidante de café en una concentración de $0,5 \mathrm{mg} / \mathrm{ml}$ (MC, I3) indujeron un incremento significativo de la secreción de serotonina $(\mathrm{p}<0,05)$ en las células Caco-2 diferenciadas. Los valores de serotonina inducida por el nuevo ingrediente alimentario $(762,9 \pm 81,4 \%)$ fueron siete veces superiores que el basal. En nuestro conocimiento, esta es la primera vez que se describe el efecto de los compuestos bioaccesibles tras la digestión oral gastrointestinal de fibra antioxidante de café sobre hormonas de saciedad. Estos resultados están en concordancia con los descritos en la literatura para el café. Estudios previos asocian al consumo de café una mejora de secreción de serotonina por las células enteroendócrinas gastrointestinales (Bakuradze, et al., 2014). Por otra parte, a los extractos de café, en concreto a los ácidos gálico y cafeico, se les atribuye potencial para incrementar la biodisponibilidad de triptófano necesario para la síntesis de serotonina mediante la inhibición de la enzima indolamina 2,3-dioxigenasa implicada en el metabolismo del triptófano (Gostner, et al., 2015). Los resultados sugieren que la inclusión de una dosis controlada de fibra antioxidante de café en la formulación del alimento podría favorecer la sensación de plenitud postprandial.

El edulcorante natural hipocalórico stevia (I4) mostró ser inefectivo en la estimulación de la producción de serotonina en las células Caco-2 diferenciadas a las concentraciones ensayadas. En un estudio de intervención llevado a cabo por Anton et al. (2010) se halló que la ingesta de stevia provocó niveles de saciedad similares a la ingesta de sacarosa. No se han encontrado más trabajos en la literatura científica en relación al efecto de la stevia pura en la secreción de esta hormona. En consecuencia, se requieren más estudios para elucidar el papel de este edulcorante hipocalórico y sus metabolitos en la secreción de serotonina. Estos resultados y los descritos en el párrafo anterior indican claramente la necesidad de realizar estudios de intervención nutricional en humanos para conocer el grado de saciedad que causa el nuevo alimento desarrollado en esta investigación. Sin embargo, los resultados sugieren un potencial efecto saciante que podría ser igual o superior al que se logra tras la ingesta de galletas preparadas según la formulación tradicional, empleando sacarosa como edulcorante.

La mayor parte de la serotonina liberada por el organismo se encuentra en las células enterocromafines del tracto gastrointestinal, que responden a los estímulos mecánicos y químicos mediante liberación de la hormona tras la ingesta de alimento (Voigt y Fink, 2015). Por tanto, es esperable que la fibra antioxidante del café, que se utiliza como ingrediente alimentario en este trabajo, pueda ejercer un mayor efecto saciante in vivo que el que aquí se describe. En esta investigación solo se ha evaluado el efecto de aquellos compuestos que quedan bioaccesibles tras su digestión gastrointestinal. No se tiene en cuenta el efecto físico de estimulación hormonal del material indigestible que podría también jugar un papel relevante en la secreción de serotonina. 
El análisis de la composición nutricional de los digeridos de galletas obtenidos (datos no mostrados) sugieren la presencia de otros compuestos que pueden estimular la secreción de serotonina, tales como azúcares. El consumo de alimentos con elevado índice glicémico (Herrera, et al., 2011; Zelkas, et al., 2015) se ha asociado a un incremento de producción de serotonina por las vesículas intestinales celulares. Los péptidos que se liberan durante el proceso de digestión del gluten que compone la harina de trigo pueden estimular principalmente la liberación de serotonina hipotalámica y cortical (Choi, et al., 2009, 2011). Por otra parte, los compuestos fenólicos presentes en la fibra dietética antioxidante podrían desempeñar un papel fundamental en la supresión del apetito también, mediante la estimulación de esta importante hormona de señalización (Huang, et al., 2013; Liu, et al., 2015). Estudios previos indican que los productos avanzados de la reacción de Maillard tales como el Ne-carboximetil-lisina están involucrados en el proceso de regulación de saciedad por medio de la liberación de serotonina en células SH-SY5Y (Holik, et al., 2013). La reacción de Maillard es el evento químico más importante que tiene lugar durante el procesado térmico de alimentos como las galletas (Tamanna y Mahmood, 2015). Por tanto, en el caso de las galletas elaboradas empleando sacarosa como edulcorante estos productos podrían contribuir a la estimulación de la liberación de esta hormona. Sin embargo, la sustitución de sacarosa por stevia limita el progreso de la reacción de Maillard en el alimento hacia las etapas avanzadas, afectando aspectos sensoriales del alimento como el desarrollo de color (Garcia-Serna, et al., 2014). En consecuencia, puede que estos productos no contribuyan de manera significativa a lograr un efecto saciante postprandial en el alimento elaborado según la nueva formulación (B2). Se requiere de más estudios para identificar los componentes alimentarios individuales que contribuyen a la liberación de la hormona tanto en el alimento elaborado siguiendo la formulación tradicional como en la propuesta en este trabajo.

\section{Liberación de GLP-1}

Se observó una secreción de GLP-1 significativa $(\mathrm{p}<0,05)$ por estimulación de las células HuTu-80 con la fracción bioaccesible de los alimentos digeridos (Tabla 2). La cantidad de hormona GLP-1 liberada en comparación con las células control no tratadas se incrementó significativamente $(\mathrm{p}<0,05)$ tras la incubación con las fracciones bioaccesibles $(0,5 \mathrm{mg} /$ $\mathrm{ml}$ ) de la galleta con alto contenido en fibra y sin azúcares añadidos (B2) $(277,9 \pm 14,1 \%)$. No se detectaron diferencias $(\mathrm{p}>0,05)$ entre la galleta control (A) y la elaborada con la nueva formulación (B2). La mínima concentración testada $(0,01 \mathrm{mg} / \mathrm{ml})$ del digerido de fibra antioxidante de café (I3) presentó valores similares de GLP-1 (p>0,05) $(269,89 \pm 17,3 \%)$ a aquellos encontrados en las galletas elaboradas con la nueva formulación (B2, 0,05 mg/ml). Es la primera vez que se describe el efecto estimulador de la secreción de GLP-1 para la fibra antioxidante de café obtenida a partir de MC generados durante el proceso de la elaboración industrial de café instantáneo. Estos resultados concuerdan con los descritos para café en células, animales (Fujii, et al., 2015) y humanas (Jokura, et al., 2015).

En algunos casos asocian la estimulación de la producción de GLP-1 del café al compuesto fenólico mayoritario de la bebida, ácido clorogénico (McCarty, 2005; Rafferty, et al., 2011). El análisis del digerido de la fibra antioxidante de café procedente de los MC (datos no mostrados) indicó gran contribución de los compuestos fenólicos sobre la capacidad antioxidante, por lo que previsiblemente el ácido clorogénico estaría presente. La stevia (I4) resultó inefectiva a las tres concentraciones ensayadas, lo que apoya los resultados obtenidos en modelo in vivo animal por Fujita et al. (2009).

La fracción bioaccesible obtenida por digestión de la nueva galleta (B2) posiblemente contiene fibra soluble (FOS). Son varios los investigadores que han descrito la existencia de una relación entre estos hidratos de carbono y la secreción de la hormona GLP-1 (Delzenne, et al., 2007; Grover, et al., 2011; Habib, et al., 2011; Li, et al., 2012), destacando el potencial de estos compuestos para promover la secreción endógena de GLP-1 y conseguir así posible prevención y/o tratamiento de la obesidad y la diabetes tipo 2. La presencia de proteínas y péptidos en los digeridos analizados también exhibir estimulación de la hormona GLP-1, confirmado ya por otros autores previamente, quienes reportaron que proteínas de trigo intactas y digeridas conseguían influir fuertemente sobre la saciedad y el efecto anti-diabético a través de la estimulación de GLP-1 (Geraedts, et al., 2010, 2011).

\begin{tabular}{|c|c|c|c|c|c|c|}
\hline & \multicolumn{3}{|c|}{ Serotonina T/C [\%] } & \multicolumn{3}{|c|}{ GLP-1 T/C [\%] } \\
\hline & $0,5 \mathrm{mg} / \mathrm{ml}$ & $0,05 \mathrm{mg} / \mathrm{ml}$ & $0,01 \mathrm{mg} / \mathrm{ml}$ & $0,5 \mathrm{mg} / \mathrm{ml}$ & $0,05 \mathrm{mg} / \mathrm{ml}$ & $0,01 \mathrm{mg} / \mathrm{ml}$ \\
\hline A & $468,3 \pm 52,6$ & $118,4 \pm 15,0 b$ & $128,52 \pm 15,3^{b}$ & $31,2 \pm 2,95 \mathrm{a}$ & $311,4 \pm 11,6^{b}$ & $157,0 \pm 9,3 c$ \\
\hline B2 & $354,7 \pm 42,7 \mathrm{a}$ & $132,6 \pm 18,9 \mathrm{~b}$ & $106,51 \pm 13,5^{b}$ & $30,3 \pm 3,97$ a & $277,9 \pm 14,1^{b}$ & $129,5 \pm 6,8^{c, d}$ \\
\hline I3 & $762,9 \pm 81,4^{c}$ & $111,9 \pm 10,6^{b}$ & $95,11 \pm 5,5^{b}$ & $21,27 \pm 2,84$ a & $193,3 \pm 14,2 \mathrm{e}$ & $269,9 \pm 17,2$ \\
\hline I4 & $95,0 \pm 13,6 b$ & $81,2 \pm 10,3 b$ & $95,85 \pm 9,1 \mathrm{~b}$ & $98,5 \pm 4,9 \mathrm{~d}$ & $106,9 \pm 3,3 \mathrm{~d}$ & $106,8 \pm 4,2 \mathrm{~d}$ \\
\hline
\end{tabular}

Tabla 2. Liberación de serotonina y GLP-1, [\%] frente al control (células no tratadas, 100\%) por estimulación de células Caco-2 y HuTu-80 con las fracciones bioaccesibles de los digeridos de galleta control a base de sacarosa (A), de galleta con stevia, fibra antioxidante de café y FOS (B2), fibra antioxidante de café (I3) y stevia pura (I4), en concentraciones de 0,5 , 0,05 y $0,01 \mathrm{mg} / \mathrm{ml}$. Los resultados se expresan como la media \pm SEM $(\mathrm{n}=3$ sub-cultivos $\times 6$ réplicas de cada uno $=18)$. Las letras diferentes indican la existencia de diferencias significativas entre los valores de todas las muestras analizadas para cada hormona en particular (ANOVA de una vía $(\mathrm{p}<0,05)$ ). 
Asimismo, el aceite de girasol empleado en las formulaciones de galletas ha demostrado tener efecto sobre la producción de GLP-1 intestinal en ensayos clínicos (Frost, et al., 2003; Manning, et al., 2013), lo que podría estar contribuyendo también al contenido total de GLP-1 liberado por células $\mathrm{HuTu}-80$ tras el tratamiento con la galleta con alto contenido en fibra y sin azúcares (B2).

\section{Conclusiones}

Por primera vez se ha descrito el efecto de la fibra antioxidante obtenida a partir de marros o posos de café (fibra antioxidante de café) en la estimulación de la secreción de las hormonas reguladoras de la saciedad, serotonina y GLP-1. Se ha logrado obtener un snack saludable con alto contenido en fibra, bajo contenido en azúcares glicemiantes, con potencial para mejorar la tolerancia a la glucosa y efecto saciante. El nuevo alimento puede ser por tanto considerado adecuado para su incorporación en una dieta destinada a la prevención y/o tratamiento de enfermedades crónicas tales como diabetes tipo 2 y obesidad. Es necesaria la realización de más estudios para elucidar la contribución de cada uno de los componentes del nuevo alimento a la secreción de serotonina y de GLP-1 y evaluar mediante estudio de intervención nutricional la magnitud de su efecto en la regulación de la saciedad, el apetito y la glucosa sanguínea postprandial en individuos sanos y enfermos. Los efectos observados de inducción de secreción de hormonas de saciedad pueden deberse a un efecto sinérgico de varios de sus ingredientes y los metabolitos que se generan durante su proceso de digestión.

\section{Reconocimientos}

Esta investigación ha sido financiada por el proyecto AGL2014-57239-R y realizada en colaboración con la Universidad de Viena. Martínez-Sáez, N. agradece a la Universidad Autónoma de Madrid la beca predoc-FPI y la ayuda para estancia breve en la Universidad de Viena. Los autores agradecen a BENEO-ORAFTI la donación de los FOS, a HELM IBERICA, S. A. de maltitol, y a PROSOL por el suministro de la materia prima para obtener la fibra antioxidante de café.

\section{Referencias}

Anton, S, Martin, C, Han, H, Coulon, S, Cefalu, W, Geiselman, P y Williamson, D, 2010. Effects of stevia, aspartame, and sucrose on food intake, satiety, and postprandial glucose and insulin levels. En: Appetite, 55(1), pp.37-43.

Bakuradze, T, Parra, G, Riedel, A, Somoza, V, Lang, R, Dieminger, N y Richling, E, 2014. Four-week coffee consumption affects energy intake, satiety regulation, body fat, and protects DNA integrity. En: Food Research International, 63, pp.420-427.

Choi, S, DiSilvio, B, Fernstrom, M y Fernstrom, J, 2009. Meal ingestion, amino acids and brain neurotransmitters: Effects of dietary protein source on serotonin and catecholamine synthesis rates. En: Physiology and Behavior, 98(1-2), pp.156-162.
Choi, S, DiSilvio, B, Fernstrom, M y Fernstrom, J, 2011. The chronic ingestion of diets containing different proteins produces marked variations in brain tryptophan levels and serotonin synthesis in the rat. En: Neurochemical Research, 36(3), pp.559-565.

del Castillo, M., Martinez-Saez, N. y Ullate, M., 2014. Healthy bakery products with high level of dietary antioxidant fibre. Patente española. PCT/ES2014/070062.

Delzenne, N, Cani, P y Neyrinck, A, 2007. Modulation of Glucagon-like Peptide 1 and Energy Metabolism by Inulin and Oligofructose: Experimental Data. En: Journal of Nutrition, 137(11), pp.2547S-2551S.

Edwards, A y Slater, N, 2009. Protection of live bacteria from bile acid toxicity using bile acid adsorbing resins. En: Vaccine, 27(29), pp.3897-3903.

EFSA, 2014. Scientific Opinion on the substantiation of a health claim related to non-digestible carbohydrates and a reduction of post-prandial glycaemic responses pursuant to Article 13 (5) of Regulation (EC) No 1924/2006. En: EFSA Journal, 12(1), pp.1-13.

Frost, G, Brynes, A, Dhillo, W, Bloom, S y McBurney, M, 2003. The effects of fiber enrichment of pasta and fat content on gastric emptying, GLP-1, glucose, and insulin responses to a meal. En: European Journal of Clinical Nutrition, 57, pp.293-298.

Fujii, Y, Osaki, N, Hase, Ty Shimotoyodome, A, 2015. Ingestion of coffee polyphenols increases postprandial release of the active glucagon-like peptide-1 (GLP-1(7-36)) amide in C57BL/6J mice. En: Journal of Nutritional Science, 4, (e9), pp.1-9.

Fujita, Y, Wideman, R, Speck, M, Asadi, A, King, D, Webber, $\mathrm{T}$ y Kieffer, T, 2009. Incretin release from gut is acutely enhanced by sugar but not by sweeteners in vivo. En: American Journal of Physiology. Endocrinology and Metabolism, 296(3), pp.E473-E479.

Garcia-Serna, E, Martinez-Saez, N, Mesias, M, Morales, F, y del Castillo, M, 2014. Use of coffee silverskin and stevia to improve the formulation of biscuits. En: Polish Journal of Food and Nutrition Sciences, 64(4), pp.243-251.

Geraedts, M, Troost, F, Fischer, M, Edens, L y Saris, W, 2011. Direct induction of CCK and GLP-1 release from murine endocrine cells by intact dietary proteins. En: Molecular Nutrition and Food Research, 55(3), pp.476-484.

Geraedts, M., Troost, F, Tinnemans, R, Söderholm, J, Brummer, R y Saris, W, 2010. Release of satiety hormones in response to specific dietary proteins is different between human and murine small intestinal mucosa. En: Annals of Nutrition and Metabolism, 56(4), pp.308-313.

Gostner, J, Schroecksnadel, S, Jenny, Klein, A, Ueberall, F, Schennach, H y Fuchs, D, 2015. Coffee Extracts Suppress Tryptophan Breakdown in Mitogen-Stimulated Peripheral Blood Mononuclear Cells. En: Journal of the American College of Nutrition, 34(3), pp.212-223.

Grover, G, Koetzner, L, Wicks, J, Gahler, R, Lyon, M, Reimer, R y Wood, S, 2011. Effects of the soluble fiber complex PolyGlycopleX $\mathrm{X}^{\oplus}\left(\mathrm{PGX}^{\oplus}\right)$ on glycemic control, insulin secretion, and GLP-1 levels in Zucker diabetic rats. En: Life Sciences, 88(9-10), pp.392-399.

Habib, N, Honoré, S, Genta, S y Sánchez, S, 2011. Hypolipidemic effect of Smallanthus sonchifolius (yacon) roots on diabetic rats: Biochemical approach. En: ChemicoBiological Interactions, 194(1), pp.31-39.

Hellfritsch, C, Brockhoff, A, Stähler, F, Meyerhof, W y Hofmann, T, 2012. Human psychometric and taste receptor responses 
to steviol glycosides. En: Journal of Agricultural and Food Chemistry, 60(27), pp.6782-6793.

Herrera, C, Smith, K, Atkinson, F, Ruell, P, Chow, C, O’Connor, H y Brand-Miller, J, 2011. High-glycaemic index and -glycaemic load meals increase the availability of tryptophan in healthy volunteers. En: The British Journal of Nutrition, 105(11), pp.1601-1606.

Holik, A, Rohm, B, Somoza, M y Somoza, V, 2013. N(ع)Carboxymethyllysine (CML), a Maillard reaction product, stimulates serotonin release and activates the receptor for advanced glycation end products (RAGE) in SH-SY5Y cells. En: Food and Function, 4(7), pp.1111-20.

Hollebeeck, S, Borlon, F, Schneider, Y, Larondelle, Y y Rogez, $\mathrm{H}, 2013$. Development of a standardised human in vitro digestion protocol based on macronutrient digestion using response surface methodology. En: Food Chemistry, 138(23), pp.1936-1944.

Huang, W, Chen, Z, Wang, Q, Lin, M, Wu, S, Yan, Q y Pan, J, 2013. Piperine potentiates the antidepressant-like effect of trans-resveratrol: Involvement of monoaminergic system. En: Metabolic Brain Disease, 28(4), pp.585-595.

Ishwarya, S, y Prabhasankar, P, 2013. Fructooligosaccharide - Retention during baking and its influence on biscuit quality. En: Food Bioscience, 4, pp.68-80.

Jokura, H, Watanabe, I, Umeda, M, Hase, T y Shimotoyodome, A, 2015. Coffee polyphenol consumption improves postprandial hyperglycemia associated with impaired vascular endothelial function in healthy male adults. En: Nutrition Research, 35(10), pp.873-881.

Kumar, V, Sinha, A, Makkar, H, de Boeck, G y Becker, K, 2012. Dietary Roles of Non-Starch Polysachharides in Human Nutrition: A Review. En: Critical Reviews in Food Science and Nutrition, 52(10), pp.899-935.

Lee, C. Y, 2016. Glucagon-like Peptide-1 Formulation - the Present and Future Development in Diabetes Treatment. En: Basic and Clinical Pharmacology and Toxicology, 118, pp.173-180.

Li, P, Lin, W, Wang, Y, Peng, W, Cai, X y Su, W, 2012. Antidiabetic activities of oligosaccharides of Ophiopogonis japonicus in experimental type 2 diabetic rats. En: International Journal of Biological Macromolecules, 51(5), pp.749-755.

Liu, Y, Lan, N, Ren, J, Wu, Y, Wang, S-T, Huang, X y Yu, Y, 2015. Orientin improves depression-like behavior and BDNF in chronic stressed mice. En: Molecular Nutrition and Food Research, 59, pp.1130-1142.

Manning, P, Sutherland, W, Manning, A, de Jong, S y Berry, E, 2013. Ingestion of thermally oxidized sunflower oil decreases postprandial lipemia mainly in younger individuals. En: Nutrition Research, 33(9), pp.711-718.

Mawe, G y Hoffman, J, 2013. Serotonin Signaling in the Gastrointestinal Tract: Functions, dysfunctions, and therapeutic targets. En: Nature Reviews Gastroenterology and Hepatology, 10(8), pp.473-486.

McCarty, M, 2005. A chlorogenic acid-induced increase in GLP-1 production may mediate the impact of heavy coffee consumption on diabetes risk. En: Medical Hypotheses, 64(4), pp.848-853.

Nutritional Heart, Lung, and Blood Institute, 2013. Managing overweight and obesity in adults: systematic evidence review from the obesity expert panel. Washington: U.S Department of Health and Human Services.

Rafferty, E, Wylie, A, Elliott, C, Chevallier, O, Grieve, D y Green, B, 2011. In vitro and in vivo effects of natural putative secretagogues of Glucagon-like peptide-1 (GLP1). En: Scientia Pharmaceutica, 79(3), pp.615-621.

Unión Europea. Reglamento (CE) 1924/2006, de 20 de diciembre de 2006. Official Journal of the European Union, 30 de diciembre de 2006, No. L404/9.

Sartori da Silva, A, Haas, P, Sartori, N, Anton, A y Francisco, A, 2007. Fructoligosaccharides: active dietary fibers. En: Boletim Do Centro de Pesquisa E Processamento de Alimentos, 25(2), pp.295-304.

Sharma, R, Yadav, R, y Manivannan, E, 2012. Study of effect of Stevia rebaudiana bertoni on oxidative stress in type-2 diabetic rat models. En: Biomedicine and Aging Pathology, 2(3), pp.126-131.

Tamanna, N y Mahmood, N, 2015. Food processing and maillard reaction products: effect on human health and nutrition. En: International Journal of Food Science, 2015, pp.1-6.

Van Bloemendaal, L, IJzerman, R, Ten Kulve, J, Barkhof, F, Konrad, R, Drent, M y Diamant, M, 2014. GLP-1 receptor activation modulates appetite- and rewardrelated brain areas in humans. En: Diabetes, 63(12), pp.4186-4196.

Voigt, J y Fink, H, 2015. Serotonin controlling feeding and satiety. En: Behavioural Brain Research, 277, pp.14-31.

World Health Organization, 2014. Global satus report on noncommunicable diseases 2014 [En línea]. Geneva: WHO. [Consulta: 14 de abril de 2016] Disponible en: http://www.who.int/mediacentre/factsheets/fs311/es/\#.

Zelkas, L, Raghupathi, R, Lumsden, A., Martin, A, Sun, E, Spencer, N y Keating, D, 2015. Serotonin-secreting enteroendocrine cells respond via diverse mechanisms to acute and chronic changes in glucose availability. En: Nutrition and Metabolism, 12(1), pp.55. 